\title{
Dislocations and the critical endpoint of the melting line of vortex line lattices
}

\author{
Jan Kierfeld and Valerii Vinokur \\ Argonne National Laboratory, Materials Science Division, 9700 South Cass Avenue, Argonne, IL 60439, USA
}

(August 3, 1999)

\begin{abstract}
We develop a theory for dislocation-mediated structural transitions in the vortex lattice which allows for a unified description of phase transitions between the three phases, the elastic vortex glass, the amorphous vortex glass, and the vortex liquid, in terms of a free energy functional for the dislocation density. The origin of a critical endpoint of the melting line at high magnetic fields, which has been recently observed experimentally, is explained.

PACS numbers: 74.60.Ge, 05.70.Jk, 64.70.Dv, 64.70.Pf
\end{abstract}

Since the pioneering work [1] where the first-order flux line lattice (FLL) melting into an entangled vortex liquid (VL) was established, there has been a continuous development of our views of the vortex lattice phase diagram in high- $T_{c}$ superconductors [2]. Weak point disorder was shown to drive the vortex lattice into a vortex glass (VG) state with zero linear resistivity [3 5]. Observations of hysteretic resistivity switching and magnetization measurements [6] have supported the first order melting of very clean lattices. A crossover from the first order melting at low magnetic fields to a continuous VG-VL transition has been related to the structural transition within the vortex solid which is described 7-10 as a topological transition between the low-field elastic VG, free from topological defects [11,12 and maintaining quasi longrange translational order 13], and the high field amorphous $\mathrm{VG}$, where disorder generates proliferation of dislocations [11]. A simple picture of the vortex phase diagram has emerged where the three generic phases - VL, the high field amorphous VG, and the low field, low temperature quasi-lattice or Bragg glass (BrG) [14] - are governed by the three basic energies: the energy of thermal fluctuations, pinning, and elastic energies. The transition lines are determined by matching of any of the two basic energies, and the match of all three energies marks the tricritical point where the first-order melting terminates [9]. While this simplistic picture is supported by observations on $\mathrm{BSCCO}$, it fails to describe the YBCO phase diagram where the endpoint of the first-order melting line appears to be separated from the point where topological transition and melting line merge [15]. Furthermore, the Lindemann-criterion approach of [7] can only qualitatively estimate the location of the transitions telling nothing about the nature of the vortex states involved.

In this Letter we undertake a step towards a quantitative theory of the entire vortex lattice phase diagram based on a unified description. We propose a model where all the transitions are dislocation-mediated, and derive the free energy for an ensemble of directed dislocations as a function of the dislocation density in the presence of disorder and thermal fluctuations. Each of the experimentally observed phases is characterized by its inherent dislocation density or, equivalently, by the characteristic dislocation spacing $R_{D}$. The elastic VG is dislocation-free and has $R_{D}=\infty$. The VL can be viewed as a vortex array saturated with dislocations such that $R_{D} \sim a$, and in the amorphous VG, $R_{D} \sim R_{a}$, where $R_{a}$ is the so-called positional correlation length on which typical vortex displacements are of the order of the lattice spacing $a$ [2]. Each phase corresponds to one of the local minima in the dislocation ensemble free energy, and dislocation densities in these minima represent the equilibrium dislocation densities in the corresponding phases. The global minimum corresponds to the thermodynamically stable phase under the given conditions, phase transitions occur when two local minima exchange their role as global minimum. This mechanism for the transitions enables us to derive Lindemann-criteria both for the locations of the thermal melting line and the disorder-induced instability line of the BrG. The characteristic scale set by the mean distance between free dislocations offers a natural explanation of the critical endpoint of the first-order melting line. While at low magnetic fields $R_{a} \gg a$ and the amorphous VG appears to contain significantly less dislocations than the VL, at higher field where $R_{a}=a$ the two phases become thermodynamically equivalent and the first order melting line terminates.

A free energy for the dislocation degrees of freedom governing phase transitions is derived from the vortex lattice elasticity theory. Dislocations in the FLL can be both of screw or edge type, but in either case they are confined to the gliding plane spanned by their Burger's vector $\mathbf{b}$ and the magnetic field [16]. The single dislocation energy consists of the core energy $E_{c}$ and of the logarithmically diverging contribution from the long-range elastic strains [17]. Accordingly, the dislocation ensemble is modeled as an array of elastic lines with a long-range Coulomb-like interaction. The energy penalty for Burger's vectors with $b>a$ leads to an effective hard-core repulsion of dislocations which conspires with the planarity constraint to limit the entropy gain of dislocations. This favors directed dislocations threading the entire sample [18] rather than large dislocation loops [19]. A single directed dislocation line is parameterized by its displacement field $u_{D}(z)$ and described by the Hamiltonian

$$
\mathcal{H}_{D}\left[u_{D}\right]=\int d z\left(E_{s}+\frac{1}{2} \epsilon_{D}\left(\partial_{z} u_{D}\right)^{2}\right)
$$


where the stiffness $\epsilon_{D} \propto \ln \left(1 / k_{z} a\right)$ has a logarithmic dispersion from the long-range interaction and $E_{s}$ is the self-energy of a straight dislocation. For thermal melting, the short wavelength limit, $k_{z} \sim 1 / a$, is relevant. After rescaling $z=\tilde{z} \frac{1}{2} \sqrt{c_{44} / c_{66}}$ dislocation energies become isotropic with $\epsilon_{D}=E_{c}=c_{D} K b^{2} / 4 \pi\left(c_{D} \approx 1\right)$ and $E_{s}=E_{c}+K b^{2} / 4 \pi \ln (L / a)$ where $K=\sqrt{c_{44} c_{66}}$ is the isotropized elastic constant $\left(c_{44}\right.$ and $c_{66}$ are the tilt and shear moduli of the vortex lattice, respectively. $L$ is the system size in the transverse direction). Note that the Peierls barrier $W_{p}$ and the associated "kinking" [17] of dislocation lines can be neglected near the melting transition. It can be shown that kinks are irrelevant above the temperature $T_{k} \sim a \sqrt{W_{d} \epsilon_{d}}$. Since we find $W_{p} \lesssim 10^{-4} E_{c}$, $T_{k}$ is much lower than $T_{m}$. Therefore, the basic length scale along the magnetic field is solely set by the competition of FL tilt and shear and given by $a_{z} \approx \sqrt{c_{44} / c_{66}} / 2$ ( $a_{z} \approx a$ in the rescaled system). The free energy of a single dislocation can be readily calculated from the partition sum $Z_{D}=\int \mathcal{D} u_{D} \exp \left(-\beta \mathcal{H}_{D}\left[u_{D}\right]\right)$ by Gaussian functional integration and consists of the core energy, the long-range strains elastic energy, and the entropy term:

$$
\frac{F_{D}(L)}{L_{z}}=E_{c}+\frac{K b^{2}}{4 \pi} \ln \left(\frac{L}{a}\right)-T \frac{1}{2 a_{z}} \ln \left(1+\frac{2 \pi T a_{z}}{\epsilon_{D} a^{2}}\right)
$$

A second order phase transition due to the formation of a single dislocation is prohibited by the logarithmic divergence of its elastic energy. However, in an ensemble of dislocations, this divergence is screened out on the distance $1 / 2 \rho a$, where $2 \rho$ is the dislocation density (the ensemble is topologically neutral to avoid the accumulation of stresses). An additional entropy cost $\left(\propto \rho^{3}\right)$ comes from the steric repulsion, and the resulting free energy density then reads

$$
\begin{aligned}
f(\rho)=2 \rho & \left(E_{c}-T \frac{1}{2 a_{z}} \ln \left(1+\frac{2 \pi T a_{z}}{\epsilon_{D} a^{2}}\right)\right)+ \\
& +2 \rho \frac{K b^{2}}{4 \pi} \ln \left(\frac{1}{2 a^{2} \rho}\right)+\rho^{3} \frac{\pi^{2}}{3} \frac{T^{2} a^{2}}{\epsilon_{D}} .
\end{aligned}
$$

$f(\rho)$ can be derived in a more rigorous manner by mapping dislocations onto a quantum system of 2D Fermions with Coulomb interaction [20,21]. At high dislocation densities, the screening mechanism in (3) is purely entropic in nature and equivalent to the formation of an exchange-hole in the 2D fermionic system. A first order melting following from (3) occurs at $T_{m} \approx 1.5 E_{c} a_{z} \approx$ $0.15 \mathrm{Ka}^{3}$, which is equivalent to melting according to the Lindemann-criterion with a Lindemann-number $c_{L} \approx 0.2$; in good agreement with experimental and numerical results. At the melting transition dislocations proliferate with a high density $\rho_{m} \approx 0.3 a^{-2}$, hence the VL is saturated with dislocations. The presented scenario does not require a simultaneous proliferation of disclinations, however, the latter are likely to appear at the high dislocation densities involved.

In the presence of a random potential $V_{\text {pin }}(\mathbf{r})$, the collectively pinned dislocation-free vortex array passes through three different scaling regimes: (i) Small scales where vortex displacements $u$ are smaller than the coherence length $\xi$ and perturbation theory applies [23]. (ii) Intermediate scales where $\xi \lesssim u \lesssim a$ and disorder potentials seen by different FLs are effectively uncorrelated. This regime is captured in so-called random manifold (RM) models [2,14, leading to a roughness $\tilde{G}(\mathbf{r})=\overline{\left\langle(\mathbf{u}(\mathbf{r})-\mathbf{u}(0))^{2}\right\rangle} \approx 4(a / 2 \pi)^{2}\left(r / R_{a}\right)^{2 \zeta_{R M}}$ where $\zeta_{R M} \approx 1 / 5$ for the $d=3$ dimensional RM with two displacement components. The crossover scale to the asymptotic behaviour is the positional correlation length $R_{a}$ where the average displacement is of the order of the FL spacing: $u \approx a / 2 \pi$ [22]. (iii) The asymptotic Bragg glass regime where the $a$-periodicity of the FL array becomes important for the coupling to the disorder and the array is effectively subject to a periodic pinning potential with period $a$ 13]. Here the logarithmic roughness $\tilde{G}(\mathbf{r}) \approx 4(a / 2 \pi)^{2} \ln \left(e r / R_{a}\right)$, i.e., $\zeta_{B r G}=\mathcal{O}(\log )$ 13, 14 takes over.

In a disordered system at $T=0$ the mechanism for dislocation proliferation is fundamentally different from the thermal melting discussed before. While thermal melting is driven by the entropy gain from unbinding dislocations, at $T=0$ the FLL benefits energetically from adjusting itself to the disorder, and dislocation proliferation leads to disorder energy gain through the dislocation degrees of freedom. It has been shown in Refs. [11, 12 that the $\mathrm{BrG}$ phase in $3 \mathrm{D}$ is stable with respect to dislocation formation. Instabilities arise, however, from the sub-asymptotic regimes [in Ref. [11] this has been partly taken into account by considering displacements in the dislocation core]. To handle analytical difficulties and to provide a unified treatment through all scaling regimes, we develop an approach to the 3D problem based on an effective random stress model which has the same displacement correlations as the full non-linear disordered model but allows for a separation of dislocation and elastic degrees of freedom. This idea is motivated by the renormalization group (RG) for the $2 \mathrm{D} \mathrm{BrG}$ which explicitly shows it renormalizes asymptotically into a random stress model [26] and has been used in Ref. 24 to show the instability of the $2 \mathrm{D} \mathrm{BrG}$ with respect to dislocations. For simplicity we consider a uniaxial model (in the incompressible limit $c_{11} \gg c_{66}$ ) which yields the same dislocation energetics as the isotropized two-component model. The Hamiltonian is

$$
\mathcal{H}[\mathbf{u}]=\int_{\mathbf{r}}\left\{\frac{1}{2} K(\nabla u)^{2}+\sigma \cdot \nabla u\right\}
$$

where $\sigma(\mathbf{r})$ is the random stress field which we assume to be Gaussian distributed with a second moment $\overline{\sigma_{i}(\mathbf{k}) \sigma_{j}\left(\mathbf{k}^{\prime}\right)}=\delta_{i j} \Sigma(k)(2 \pi)^{3} \delta\left(\mathbf{k}+\mathbf{k}^{\prime}\right)$ characterized by the function $\Sigma(k)$ in Fourier space. The effective random stresses causing displacements with the same (2-point) correlations as those for the $\mathrm{RM}$ or $\mathrm{BrG}$ regime are 


$$
\Sigma(k)= \begin{cases}\mathrm{BrG}: & \frac{1}{2} K^{2} k^{-1} a^{2} \\ \mathrm{RM}: & B_{1 / 5} K^{2} k^{-1} a^{2}\left(k R_{a}\right)^{-2 / 5}\end{cases}
$$

with a numerical constant $B_{1 / 5}$ depending only on the roughness $\zeta_{R M}=1 / 5$ (the exact crossover between the two regimes is non-trivial [14]). The validity of the random stress model is well-established in 2D. Besides, the functional RG treatment of the $\mathrm{BrG}$ in $d=4-\epsilon$ dimensions shows that displacements asymptotically obey Gaussian statistics up to the first order in $\epsilon$ [27], which can always be modeled by an effective random stress field. Similarly, in a real-space RG analysis [28], the relevant fixed point is perturbative in $\epsilon$ and an analogous argument applies.

Starting from (伍) the energy for an ensemble of curved dislocation lines with a Burger's vector density $\mathbf{b}(\mathbf{r})$ in the disordered system can be calculated (for example along the lines of Ref. [25]). In the random stress model the Hamiltonian decouples into the elastic part and a dislocation part:

$$
\mathcal{H}_{D}[\mathbf{b}]=\int_{\mathbf{r}} \int_{\mathbf{r}^{\prime}} \frac{K}{2} \mathbf{b}_{\mathbf{r}} \cdot \mathbf{b}_{\mathbf{r}^{\prime}} G_{0}\left(\mathbf{r}-\mathbf{r}^{\prime}\right)+\int_{\mathbf{r}} \mathbf{b}_{\mathbf{r}} \cdot \mathbf{g}_{\mathbf{r}}
$$

where $G_{0}(\mathbf{r})=1 /(4 \pi r)$ is the 3D Green's function. Here $\mathbf{g}(\mathbf{r})$ is an effective random potential for dislocations lines defined by the transversal part of $\sigma$ through $\nabla \times \mathbf{g}=\sigma_{T}$ (cf. 24]). This energy contains the long-range elastic energy $E_{s}$ of dislocations in the first term and in the stochastic second term the disorder energy $E_{d i s}$ of the dislocation which allows dislocations to gain energy by optimizing their paths. The disorder energy is completely determined by the FL displacement correlations through $\overline{g_{i}(\mathbf{k}) g_{j}\left(\mathbf{k}^{\prime}\right)}=\delta_{i j} K \Sigma(k) k^{-2}(2 \pi)^{3} \delta\left(\mathbf{k}+\mathbf{k}^{\prime}\right)$ in the different regimes given by (5). For a single directed dislocation line, the Hamiltonian (6) reduces to the problem of a directed elastic line (with a logarithmic dispersion, see above) in a random potential that is long-range correlated due to (5). For a directed dislocation of length $L_{z}$ and confined in the transversal direction to a scale $L$, the elastic energy is again given by (2) with $T=0$. The mean square disorder energy fluctuations are

$$
\overline{E_{d i s}^{2}\left(L_{z}, L\right)}= \begin{cases}\text { BrG: } & c_{B r G} E_{D}^{2} L_{z} L \\ \mathrm{RM}: & c_{R M} E_{D}^{2} L_{z} L\left(\frac{L}{R_{a}}\right)^{2 / 5}\end{cases}
$$

with numbers $c_{B r G}, c_{R M}=\mathcal{O}(1)$. These expressions give an estimate of the typical disorder energy a dislocation line can gain. They neglect rare fluctuations in the energy gain from optimally positioning the dislocation in the transversal plane which give logarithmic corrections $\sim$ $\mathcal{O}(\ln L)$ [12]. The optimal path of the dislocation will be rough $u_{D} \sim L_{z}^{\zeta_{D}}$ with an exponent $\zeta_{D}$. The roughness can be obtained by a Flory argument that equates the elastic energy from the deformation $\epsilon_{D}(L) u_{D}^{2} / L$ (with a logarithmically dispersive $\epsilon_{D} \sim \ln L$ on large scales) and the disorder energy ${\overline{E_{d i s}^{2}\left(L_{z}=L, L\right)}}^{1 / 2}$ on one large length scale set by the fluctuation wavelength $L$. This yields $\zeta_{D}(B r G)=1-\mathcal{O}\left(\log ^{1 / 2}\right)$ and $\zeta_{D}(R M)=\frac{11}{10}-\mathcal{O}\left(\log ^{1 / 2}\right)$ where logarithmic corrections come from the dispersion of the stiffness and rare fluctuations. Since $\zeta_{D}(B r G) \leq 1$, the BrG appears to be marginally stable against penetration of a single directed dislocation whereas $\zeta_{D}(R M)>1$ such that the random manifold is clearly unstable. Note that the scaling arguments of Ref. [12] taking into account rare fluctuations give the same result as the simple Flory argument neglecting rare fluctuations. We argue that the sub-asymptotic instability of the FLL in the RM regime on scales $L<R_{a}$, combined with the asymptotic stability in the $\mathrm{BrG}$ regime for scales $L>R_{a}$, leads to a disorder-induced dislocation proliferation via a weak first order phase transition. The characteristic dislocation density $\rho_{c} \sim R_{a}^{-2}$ at the transition is given just by the crossover scale $R_{a}$. The discontinuities in this transition are small and may eventually disappear if the length scale $R_{a}$ becomes of the order of typical sample dimensions. Note also that, qualitatively, this result is based only on the fact that the instability sets in within a sub-asymptotic regime; the random stress model is only used to quantify our findings. It enables us to estimate typical free energy minima for ensembles of dislocation lines with rough optimized paths at $T=0$. The screened long-range elastic energy density for a (neutral) dislocation ensemble with density $2 \rho$ is given by $e_{D}(\rho)=2 \rho\left(E_{D}+\left(K b^{2} / 4 \pi\right) \ln \left(1 / \sqrt{2} a \rho^{1 / 2}\right)\right)$ as in (3) at $T=0$. Dislocations are confined to a transversal scale $R_{D} \simeq \rho^{-1 / 2}$ set by the distance to the next dislocation, hence they optimize their disorder energy gain on each longitudinal scale $L_{z} \simeq R_{D}$ independently. Using (7) with $L_{z}=L=\rho^{-1 / 2}$ for the $\operatorname{BrG}$ regime $\left(\rho<R_{a}^{-2}\right)$ and the RM regime $\left(\rho>R_{a}^{-2}\right)$, we can estimate the corresponding minimal free energy densities (not considering logarithmic corrections from rare fluctuations)

$$
f(\rho) \approx e_{D}(\rho)-\left\{\begin{array}{l}
\mathrm{BrG}: 2 A_{B r G} E_{c} \rho \\
\mathrm{RM}: 2 A_{R M} \frac{E_{c}}{a^{2}}\left(\rho a^{2}\right)^{\frac{9}{10}}\left(\frac{a}{R_{a}}\right)^{\frac{1}{5}}
\end{array}\right.
$$

with numbers $A_{B r G}, A_{R M}=\mathcal{O}(1)$. When both results in (8) are combined one indeed finds a local minimum in the free energy density at $\rho \approx R_{a}^{-2}$ that characterizes an amorphous VG phase. Over a wide range of magnetic fields the dislocation density in the amorphous $\mathrm{VG}$ is much lower than in the VL for which we have found $\rho \approx 0.3 a^{-2}$ above. The elastic BrG phase looses stability with respect to dislocation proliferation and a transition into an amorphous VG phase if the local minimum at $\rho \approx R_{a}^{-2}$ becomes the global free energy minimum. This occurs via a weak first order transition when $R_{a} / a=C$ with a "Lindemann-number" $C=\mathcal{O}(10)$. This is identical to the Lindemann criterium obtained in Refs. 11, 10 including the numerical value of $C$ [22]. As it was shown in Ref. [10] it is equivalent to a Lindemanncriterion $\overline{\left\langle(u(a)-u(0))^{2}\right\rangle}=c_{L}^{2} a^{2}$ in its more familiar form (see [7]) with $c_{L}=\frac{1}{2 \pi} C^{-1 / 5} \approx 0.1$. 


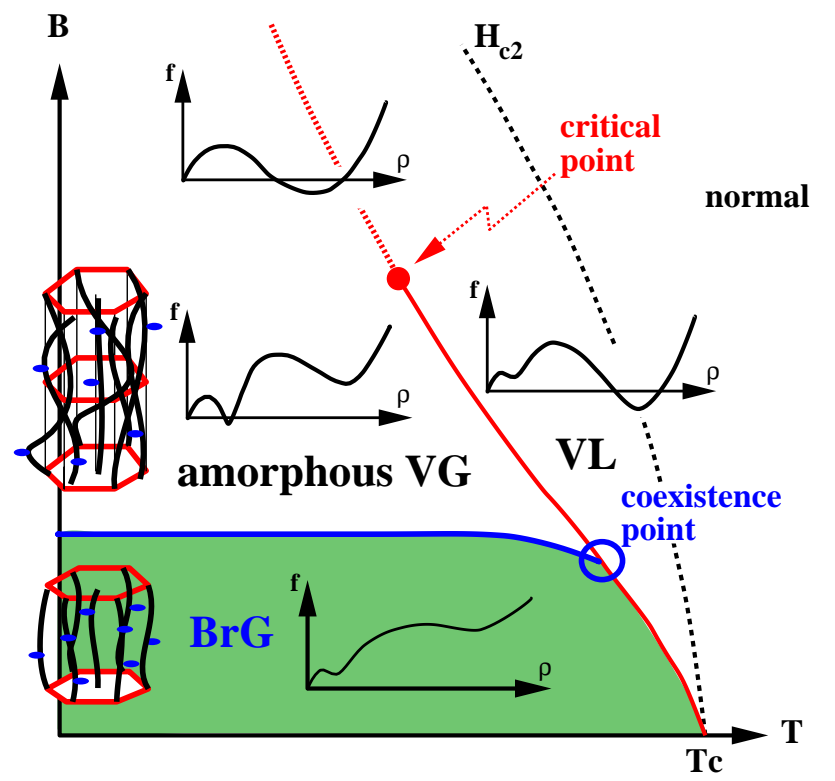

FIG. 1. Schematic phase diagram of YBCO. Insets show typical free energy densities $f$ of a dislocation ensemble as function of the dislocation density $\rho$.

We have identified three possible minima for the free energies (3) and (8): (i) The dislocation-free minimum at $\rho=0$ which is stable in the elastic BrG phase at low $T$ and low $H$. (ii) The minimum at $\rho \sim a^{-2}$ that becomes stable in the disorder-free case for high $T$ in the VL. (iii) A minimum at $\rho \sim R_{a}^{-2}$ which is realized in the amorphous VG. Combining our results for the thermal melting and the disorder-induced "melting", we have obtained a qualitative theory for the entire phase diagram of the vortex matter. The experimentally observed critical endpoint of the first order melting line finds a natural explanation. At elevated fields the positional correlation length $R_{a}$ decreases 10$]$ and finally reaches $R_{a} \sim a$ such that the two free energy minima of the VL and the amorphous VG merge. Both these phases become thermodynamically indistinguishable and have identical equilibrium lattice order. Above the critical endpoint there might still exist a dynamic transition (or crossover) which involves the thermal depinning of dislocations, similar to the well-known thermal depinning transition of, for example, a single pinned vortex line. The critical endpoint is located at the magnetic induction determined by the condition $R_{a} \approx 2 a$ (which is again equivalent to a Lindemann criterion but with $C_{c p} \approx 2$ or $c_{L} \approx 0.14$ ). This gives the estimate $B_{c p} / H_{c 2} \approx(2 \pi)^{-13 / 3}(\delta / \varepsilon)^{-10 / 9} C_{c p}^{-16 / 15} \sim$ $\mathcal{O}\left(10^{-1}\right)$ where we followed Ref. [2] and introduced a dimensionless disorder strength $\delta / \varepsilon \sim 10^{-3}$ for high- $T_{c}$ materials (cf. [10]). This value is approximately a factor 10 higher than the instability field $B_{B r G}$ of the BrG and thus the "coexistence point" where the topological transition line ends in the first order melting line and all three phases - elastic BrG, amorphous VG, and VL - can coexist.

We thank A.E. Koshelev, H. Nordborg, and D. Lopez for stimulating discussions, M. Kardar for bringing the article of T. Bohr to our attention, and D. Blair for a critical reading of the manuscript. This work was supported by Argonne National Laboratory through the U.S. Department of Energy, BES Materials Sciences, under Contract No. W-31-109-ENG-38, and by NSF-STC under Contract No. DMR91-20000 STcS. J. K. acknowledges support from the Deutsche Forschungsgemeinschaft under Grant No. KI 662/1.

[1] D.R. Nelson, Phys. Rev. Lett. 60, 1975 (1988).

[2] G. Blatter, M.V. Feigelman, V.B. Geshkenbein, A.I. Larkin, and V.M. Vinokur, Rev. Mod. Phys. 66, 1125 (1994).

[3] M.V. Feigelman et al, Phys. Rev. Lett. 63, 2303 (1989).

[4] M.P.A. Fisher, Phys. Rev. Lett 62, 1415 (1989).

[5] R.H. Koch et al., Phys. Rev. Lett. 63, 1511 (1989).

[6] R. Liang et al., Phys. Rev. Lett. 76, 835 (1996); U. Welp et al., Phys. Rev. Lett. 76, 4809 (1996); E. Zeldov et al., Nature 375, 373 (1995).

[7] D. Ertaş and D.R. Nelson, Physica C 272, 79 (1996).

[8] A.E. Koshelev and V. Vinokur, Phys. Rev. B 57, 8026 (1998).

[9] V. Vinokur et al., Physica C 295, 209 (1998).

[10] J. Kierfeld, Physica C 300, 171 (1998).

[11] J. Kierfeld, T. Nattermann, and T. Hwa, Phys. Rev. B 55, 626 (1997).

[12] D.S. Fisher, Phys. Rev. Lett. 78, 1964 (1997).

[13] T. Nattermann, Phys. Rev. Lett. 64, 2454 (1990).

[14] T. Giamarchi and P. Le Doussal, Phys. Rev. B 52, 1242 (1995).

[15] D. Lopez et al., Phys. Rev. Lett. 80, 1070 (1998).

[16] D. Nelson and C. Marchetti, Phys. Rev. B 41, 1910 (1990).

[17] J.P. Hirth and J. Lothe, Theory of Dislocations (McGraw-Hill, New York, 1968).

[18] M.-C. Miguel and M. Kardar, Phys. Rev. B 56, 11903 (1997).

[19] It has been shown that small dislocation loops are always relevant but do not chamge the transition temperature. [T. Bohr, Phys. Rev. B 25, 6981, 1982]. They only slightly modify the first order melting transition under consideration [21]. Note also the difference to the 3D XY model which is known to have a second order phase transition mediated by unconstrained vortex-loops.

[20] T. Yamamoto and T. Izuyama, J. Phys. Soc. Jpn. 57, 3742 (1988).

[21] J. Kierfeld and V. Vinokur, to be published.

[22] Note the difference by factors of $2 \pi$ in the definition of $R_{a}$ as compared to Ref. 110].

[23] A.I. Larkin, Sov. Phys. JETP 31, 784 (1970).

[24] C. Zheng, P.L. Leath, and D.S. Fisher, Phys. Rev. Lett. 82, 1935 (1999).

[25] M.S. Li et al., Phys. Rev. B 54, 16024 (1996).

[26] J.L. Cardy and S. Ostlund, Phys. Rev. B 25, 6899 (1982).

[27] T. Emig, Ph.D. thesis, Cologne University, 1998.

[28] J. Villain and J.F. Fernandez, Z. Phys. B 54, 139 (1984). 